\title{
Ensino de Lógica de Programação no Ensino Médio e suas implicações na Neurociências
}

\author{
Narúsci S. Bastos ${ }^{1}$, Diana F. Adamatti ${ }^{1}$, Fernanda A. H. de Carvalho ${ }^{2}$ \\ ${ }^{1}$ Porgrama de Pós-graduação em Computação - Universidade Federal do Rio Grande (FURG) \\ S/CEP - Rio Grande - RS - Brasil \\ ${ }^{2}$ Programa Pós-Graduação Educação em Ciências: Química da Vida e Saúde \\ Universidade Federal do Rio Grande (FURG) - S/CEP - Rio Grande - RS - Brasil \\ \{naruscibastos, dianaada, fahc.rg\}@gmail.com
}

\begin{abstract}
This paper proposes a study on the development of logic skills in high school students, based on Neuroscience. The aim is to identify possible changes in brain physiology pre and post computational logic training and characterize brain activity during the resolution of the logic test problems. To carry out this study, it was necessary to collect brain signals in two stages: before the computational logic of training and after training, while individuals solved a logic test. From these data we analyze the areas with greater brain activity during the resolution of each proposed issue, which were collected through non-invasive technique using EEG equipment (electroencephalogram) and BCI (Brain Computer-interface).
\end{abstract}

Resumo. Este trabalho propõem um estudo sobre o desenvolvimento de habilidades de lógica em estudantes do ensino médio, fundamentado na Neurociência. O objetivo é identificar possíveis mudanças na fisiologia cerebral pré e pós treinamento de lógica computacional e caracterizar as atividades cerebrais durante a resolução dos problemas do teste de lógica. Para realizar este estudo, foi necessário a coleta de sinais cerebrais em dois momentos: antes do treinamento de lógica computacional e após o treinamento, enquanto os indivíduos resolviam um teste de lógica. A partir desses dados analisam-se as áreas com maior atividade cerebral durante a resolução de cada questão proposta, que foram coletados através de técnica não-invasiva utilizando um equipamento EEG (Eletroencefalograma) e BCI (Brain Computer Interface).

\section{Introdução}

A neurociência estuda o funcionamento, desenvolvimento e estrutura do sistema nervoso. Comumente tratada como um ramo da biologia, vem se tornando uma ciência interdisciplinar, contribuindo com campos como medicina, educação, química e computação.

De acordo com Gazzaniga e Heatherton (2005), "O pensamento atual argumenta que a maneira pela qual o pensamento do adolescente se desenvolve depende muito do conteúdo dos problemas que os adolescentes encontram e dos contextos em que se encontram." daí a importância de oferecer atividades de intervenção. A tecnologia tem oferecido diversas ferramentas para que os jovens possam exercitar habilidades, como a lógica, que tem papel fundamental para a capacidade de resolver problemas encontrados no dia a dia. 
O objetivo principal deste trabalho é investigar possíveis contribuições do treinamento de lógica computacional para o desenvolvimento de raciocínio lógico em estudantes do ensino médio. Para tal, foi necessário identificar possíveis mudanças na fisiologia cerebral pré e pós treinamento na área de lógica, bem como, caracterizar a atividade cerebral durante a realização do teste de lógica antes e depois do treinamento. Finalmente, se avalia a competência/habilidade lógica dos estudantes, identificando assim possíveis diferenças de gêneros ${ }^{1}$.

É importante salientar que esta pesquisa é inovadora e trabalhos relacionados ao tema não foram encontrados na literatura. Contudo, por se tratar de uma pesquisa interdisciplinar, foi necessário o estudo de trabalhos correlatos, como BCI, aprendizagem e cognição.

O trabalho é constituído de 5 seções, a seção 2 apresenta o ferramental teórico e prático utilizado neste trabalho. Já na seção 3 está a metodologia adotada. Os resultados e sua discussão estão na seção 4. Por fim, são apresentadas considerações finais sobre esta pesquisa.

\section{Ferramental Teórico e Prático}

\subsection{Cérebro e Aprendizagem}

O cérebro é a parte mais importante do sistema nervoso, pois é através dele que obtém-se consciência das informações que chegam pelos órgãos dos sentidos e processa-se essas informações, comparando-as com as vivências e as expectativas, sendo capaz de modificar os comportamentos e aprender. Há ainda os processos mentais como o pensamento, capacidade de julgamento e a atenção, que são resultados do seu funcionamento [Cosenza e Guerra, 2011]. Estas capacidades realizam-se através de circuitos nervosos conhecidos como neurônios. Um neurônio é capaz de disparar impulsos seguidamente, muitas vezes por segundo, mas as informações só podem ser transmitidas para uma outra célula, por meio do axônio, que é uma estrutura que ocorre geralmente nas porções finais do prolongamento neural. Sendo o local por onde a passagem da informação entre as células, chamado de sinapses, e a comunicação é feita através da liberação de um neurotransmissor [Cosenza e Guerra, 2011].

A neurociência cognitiva afirma que a aprendizagem e a memória estão fortemente relacionadas, sendo a memória a base orgânica para que ocorra a aprendizagem. Memorizar não é o mesmo que aprender, mas para que ocorra aprendizagem, é preciso que as informações fiquem armazenadas na nossa memória. Para Izquierdo (2004) "A memória é o processo pelo qual aquilo que é aprendido persiste ao longo do tempo".

Carvalho (2007), fundamentada em Ratey (2002) e Lent (2001), destaca que pensar, aprender e memorizar são processos biológicos realizados no cérebro, porém distintos. $\mathrm{O}$ arquivamento seletivo, a evocação de informações, envolve um conjunto de processos neurobiológicos e neuropsicológicos que caracterizam a memória. A aprendizagem é decisiva para o pensamento, o qual deriva da capacidade de lidar com as informações das áreas de associação motora, sensorial e mnemônica. Já o pensamento é o referencial para a orientação do comportamento, pois seu processamento envolve o recebimento, a

\footnotetext{
${ }^{1}$ Esta pesquisa é parte do projeto CNPq - Edital 18/2013 ( Meninas e Jovens Fazendo Ciências Exatas, Engenharias e Computação ).
} 
percepção, a compreensão, o armazenamento, a manipulação, o monitoramento e o controle essenciais para lidar com o fluxo constante de dados objetivando planejar ações.

\section{2. Áreas Cerebrais e Sistemas BCI}

O cérebro humano é dividido em dois hemisférios especializados. Sendo assim cada hemisfério é responsável por um grupo de funções, que acabam por trabalhar em conjunto. Experimentos revelaram que as especialidades dos hemisférios podem ser bem diferentes, mas dificilmente essa especialidade é exclusivamente funcional.

Para Cosenza e Guerra (2011), o hemisfério esquerdo é normalmente responsável pelo processamento da linguagem, que na maioria das pessoas é muito mais competente que o hemisfério direito na sua percepção e expressão. Para Lent (2004), o hemisfério esquerdo é também melhor na realização mental de cálculos matemáticos, no comando da escrita e na compreensão dela através da leitura. Já o hemisfério direito, é melhor na percepção de sons musicais e reconhecimento de faces.

Os anatomistas geralmente agrupam o encéfalo humano em grandes regiões, chamadas lobos, cujos limites nem sempre são precisos, mas transmitem uma ideia inicial de localização regional.

Cada uma dessas regiões apresenta funções especializadas como: O lobo occipital é fundamentalmente envolvido com o sentido da visão, está dividido em múltiplas áreas visuais distintas, na qual a maior é o córtex visual primário. O parietal é parcialmente dedicado ao sentido do tato, agrupa funções de sensibilidade corporal e reconhecimento espacial. O lobo temporal contém o córtex auditivo primário, processa dados auditivos, aspectos elaborados da visão, a compreensão linguística e alguns aspectos da memória. Finalmente, o lobo frontal que é essencial para o planejamento de ações cognitivas, memória e movimento [Gazzaniga and Heatherton 2006][Lent 2001].

Os sistemas BCI (Brain Computer Interface) surgem como ferramentas que permitem um método de comunicação baseado em atividade neural gerada pelo cérebro, sem exigir qualquer outro tipo de estímulo, como movimentos musculares criados pelos comandos cerebrais. São sistemas que utilizam sinais elétricos, detectados no escalpo da superfície cortical ou em áreas subcorticais. Seu objetivo é criar a interação entre o usuário e o dispositivo externo, como computadores, interruptores ou próteses [Machado et. al, 2009].

Atualmente, os sistemas BCI desenvolvidos são ferramentas que podem auxiliar os usuários a se comunicar e realizar atividades cotidianas, ainda que apresentem um sucesso limitado e se encontrem principalmente em ambientes de pesquisa. No entanto, tem sido desenvolvido para usuários com deficiências mentais e físicas, para pessoas que não apresentam nenhum tipo de deficiência e principalmente no ramo do entretenimento, na indústria de jogos digitais.

\subsection{Ferramenta para Coletas de Sinais Cerebrais}

A ferramenta ActiChamp ${ }^{2}$ desenvolvida por Brain Vision LLC, é sistema de amplificador modular, que integra grandes componentes finais para Análise eletrofisiológica como

\footnotetext{
${ }^{2}$ Para a coleta de sinais cerebrais foi utilizada a ferramenta Actichamp disponível em: http://www.brainvision.com/actichamp.html.
} 
EEG, BCI, entre outros. Usou-se para a coleta dos dados o módulo de 32 canais em combinação com eletrodos ActiCap, que é uma touca inserida no couro cabeludo, que possui os canais expostos no padrão 10-20 onde os 32 eletrodos são inseridos para fazer o contato com cérebro do indivíduo.

Os sinais adquiridos através dos eletrodos e sensores são amplificados, digitalizados e em seguida transferidos para um computador por entrada USB, que possibilita o armazenamento e exibição dos dados. O Acticap é conectado ao amplificador ActiChamp, fazendo assim a transmissão dos dados captados pelos eletrodos.

Para que se tenha uma melhor compreensão, a Figura 1 apresenta como são distribuídos ao longo do encéfalo e as áreas que cada grupo de eletrodo captura, bem como o nome de cada canal.

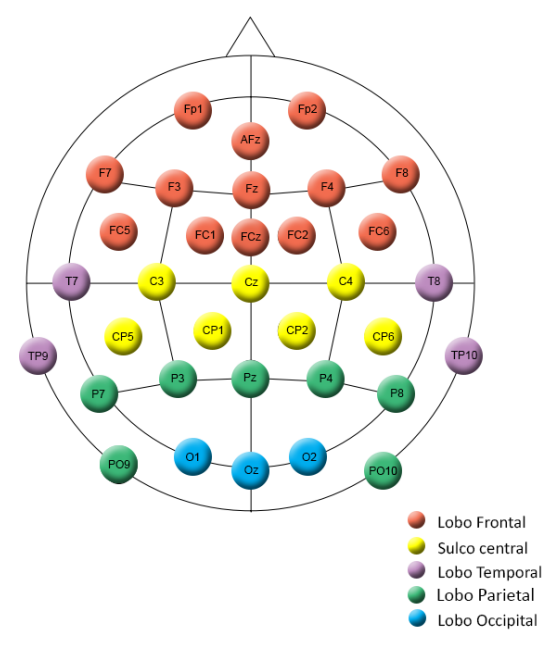

Figura 1. Localização dos eletrodos referentes aos 32 canais do Acticap (padrão internacional “10-20").

Os resultados obtidos na análise dos sinais cerebrais baseados no mapa topográfico 3D, foram validados com base na neurociência, com o auxilio de uma especialista na área. Com base na literatura, essa especialista formulou a Tabela 1, em que apresenta as regiões cerebrais, bem como os eletrodos espacialmente posicionados conforme o padrão internacional "10-20", referentes as suas funcionalidades.

Tabela 1. Áreas cerebrais, eletrodos e as funções correspondentes.

\begin{tabular}{l|l|l}
\hline Região & Eletrodo & Funções proprietárias \\
\hline Lobo Frontal & $\begin{array}{l}\text { Fp1,Fp2,AFz,F7,F3,Fz,F4, } \\
\text { F8,FC5,FC1,FCz,FC2,FC6 }\end{array}$ & Funções executivas \\
\hline Lobo Temporal & T7,TP9,T8,T10 & Percepção de movimentos biológicos \\
\hline Lobo Parietal & P7, P3, Pz, P4, P8, P9,P10 & $\begin{array}{l}\text { Percepçaõ somatossensorial, } \\
\text { representações espaciais e percepções } \\
\text { táteis. }\end{array}$ \\
\hline Lobo occipital & O1,Oz,O2 & Visualização de imagens. \\
\hline
\end{tabular}




\subsection{Capacidade Cognitiva e Aprendizagem Lógica}

No que diz respeito a habilidades linguísticas e de raciocínio, essas influenciam na aprendizagem de lógica, tanto no campo da percepção espacial quanto da compreensão linguística.

Forbellone e Ebrspacher (2005) observam que, sendo o raciocínio a forma mais complexa do pensamento, a lógica estuda a "correção do raciocínio", colocando ordem no pensamento. Tendo em vista essas ideias, nota-se a importância da lógica na vida, não só como teoria, mas como prática, uma vez que, quando um ser humano quer pensar, falar, escrever ou agir corretamente, necessita colocar "ordem no pensamento", fazendo uso da lógica.

A importância de se ter uma boa habilidade de raciocínio lógico, é fundamental para o indivíduo solucionar com maior eficiência problemas do dia a dia, que normalmente são problemas mal estruturados. Existem duas classes de problemas: bem estruturados ou bem definidos e os mal estruturados ou mal definidos [Sternberg 2012].

Os problemas bem estruturados possuem recursos claros, caminhos nítidos para a solução, como uma formula matemática. Problemas mal estruturados, não apresentam recursos claros e prontamente disponíveis para a solução. As pessoas que se propõem a solucionar esses problemas apresentam dificuldades para elaborar representações mentais apropriadas para modelar esses problemas e suas resoluções. Para tais problemas, grande parte da dificuldade consiste em elaborar um plano a fim de seguir sequencialmente uma série de passos que avancem cada vez mais próximo a solução [Sternberg 2012],[Eysenck and Keane 2007].

Quanto a influência do meio social no desenvolvimento de habilidades cognitivas, sem dúvida, o cérebro é modificável pela cultura, pois diante da plasticidade neuronal é possível reconfigurar as redes neurais e reconstruir conhecimento. Sternberg (2012) destaca que "A inteligência humana é altamente maleável. Pode ser moldada e incrementada por meio de vários tipos de intervenções". A fase da adolescência é um momento de intenso desenvolvimento cerebral.

Conforme Gazzaniga e Heatherton (2005), os adolescentes processam informações com mais rapidez, utilizam recursos de processamento com mais eficiência e tem maior flexibilidade cognitiva. Tal eficiência do controle cognitivo na adolescência coincide com mudanças biológicas no desenvolvimento cerebral. Os autores lembram que Piaget atribuía as mudanças no pensamento adolescente ao surgimento das operações formais.

\section{Metodologia}

Com intuito de investigar as possíveis contribuições do treinamento de lógica computacional para o desenvolvimento de raciocínio lógico em estudantes do ensino médio, procurou-se identificar possíveis mudanças na fisiologia cerebral pré e pós treinamento de lógica computacional, e caracterizar as atividades cerebrais durante a resolução dos problemas de um teste de lógica. Para realizar este estudo, foi necessário a coleta de sinais cerebrais em dois momentos: antes do treinamento de lógica computacional e após o treinamento.

A partir desses dados, analisou-se as áreas com maior atividade cerebral durante a 
resolução de cada questão proposta, que foram coletados através de técnica não-invasiva utilizando um equipamento EEG (ActiChamp). Estas técnicas de eletroencefalografia tem sido as mais utilizadas para a aquisição de sinais BCI.

Para se atingir esses objetivos, foram realizados os seguintes passos:

- Aplicação de questionário de perfil e teste de lógica afim de selecionar as amostras;

- Escolha do grupo de alunos que foram analisados (alunos pertencentes aos dois últimos anos do ensino médio);

- Coleta de sinais cerebrais antes do treinamento de lógica computacional;

- Oficina de ensino de lógica computacional, utilizando a linguagem Scratch, com duração de 24 horas/aulas, ministradas em 2 meses;

- Coleta dos sinais cerebrais após o termino da oficina de ensino de lógica computacional;

- Análise dos dados;

\section{Resultados e Discussão}

Para as coletas de sinais cerebrais, mediante autorização do CEPAS $^{3}$ (Comitê de Ética em Pesquisa na Área da Saúde), tanto para a coleta pré treinamento como pós treinamento, foram aplicados, individualmente, o mesmo teste de lógica para os 3 sujeitos. Os sinais cerebrais foram coletados durante a execução de cada teste de lógica. Para isso, cada sujeito teve um horário estabelecido a fim de evitar interferências, e o teste e a coleta de sinais cerebrais foram realizados em local isolado dos demais, somente com a presença da pesquisadora e um assistente. Foram formuladas duas hipóteses, uma para cada estágio da analise (pré e pós treinamento), com a intenção de verificar se as suposições são admissíveis. As imagens foram geradas em formato 3D. No qual, representam uma média de ativação para as áreas cerebrais, sendo uma informação mais confiável.

Foram proposta duas hipóteses, a primeira diz respeito a coleta pré treinamento de lógica, a segunda pós treinamento.

Hipótese 1: Os alunos sem treinamento em raciocínio lógico tendem a ativar diversas áreas cerebrais. Segundo Sternberg (2012), para a solução de problemas mal estruturados, como é o caso das questões de lógica, grande parte da dificuldade reside em elaborar um plano a fim de seguir sequencialmente uma série de passos que avancem cada vez mais próximo da solução, o que favorece erros iniciais, tomada de percursos indiretos e todos os tipos de erros. Sendo assim, o conhecimento do domínio e as habilidades de justificação provaram ser importantes para a resolução de problemas. Portanto, uma vez que o indivíduo não possui boa habilidade de raciocínio lógico, possivelmente irá apresentar dificuldades ao elaborar a sequência de passos corretos para encontrar a solução correta.

Hipótese 2: Os alunos com treinamento em raciocínio lógico ativam áreas cerebrais específicas. Em seus estudos, os autores Eysenck e Keane (2007) observam que indivíduos tendem a ativar menos áreas cerebrais após a prática de exercícios que envolvem raciocínio. Sternberg (2006), em seus achados, afirma que melhores alunos apresentam

\footnotetext{
${ }^{3}$ CAAE: 34417214.3 .0000 .5324$.
} 
maior probabilidade do que os menos capazes para dedicar mais tempo na fase inicial, decidindo como solucionar um problema, e menos tempo solucionando-o efetivamente, pois ao dedicarem mais tempo decidindo antecipadamente o que fazer, os alunos eficazes apresentam menor probabilidade de ser vítimas de inícios errados, percursos indiretos e todos os tipos de erros. Com isso, indivíduos treinados, são mais propensos a tomar caminhos mais curtos e diretos para solucionar os problemas (ditos problemas mal estruturados), ativando assim um número menor de áreas cerebrais.

Neste trabalho, apresenta-se os dados completos de um dos sujeitos analisados. A Figura 2 apresenta duas coletas, a de número 1 corresponde a coleta pré treinamento e a numero 2 a pós treinamento de lógica, como pode-se observar a figura 2 com numeração 1 mostra as atividades cerebrais executadas pelo sujeito 2 nos primeiros momentos do desenvolvimento da questão, em que foram ativadas diversas áreas além das esperadas, como o lobo frontal direito, temporal esquerdo, parietal esquerdo e sulco central direito. Já a figura 2 com numeração 2 mostra as atividades cerebrais executadas pelo sujeito 2 nos primeiros momentos do desenvolvimento da questão, mostra que foram ativadas somente as áreas necessárias para a execução do exercício. Pode-se observar que os lobos frontal, temporal esquerdo, parietal e occipital mostram maior atividade.Pode-se observar que indivíduo ativou todas as áreas necessárias para o desenvolvimento da questão, e nenhum área inesperada.
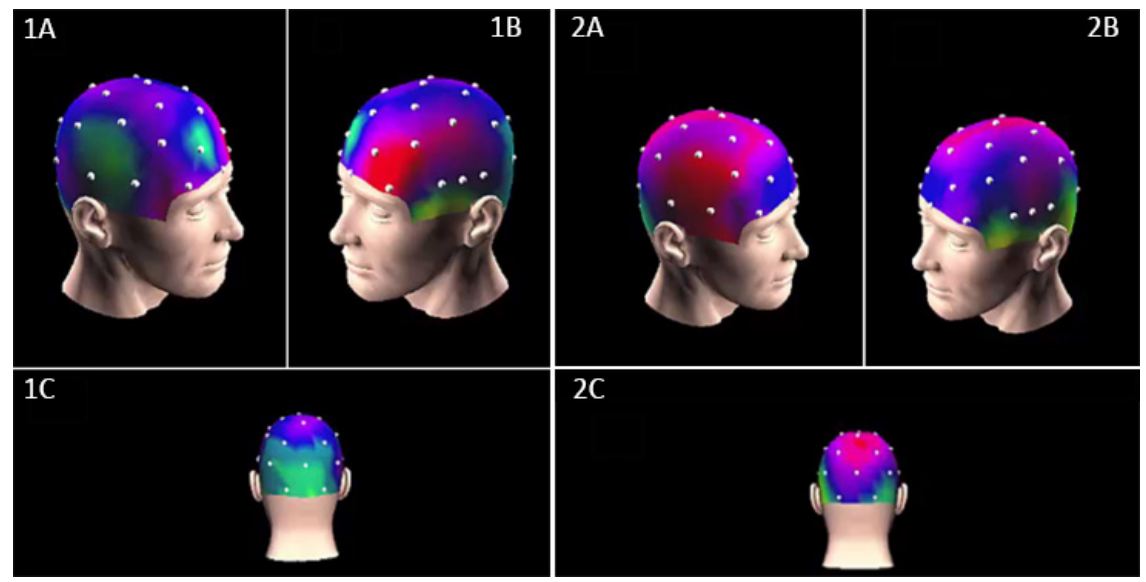

Figura 2. Principais áreas ativadas pelo sujeito durante a execução dos exercícios. As imagem numerada com 1 corresponde a análise pré treinamento, a numero 2 à coleta pós treinamento. Para as letras, A: Mapa topográfico 3D correspondente ao hemisfério direito $B$ : Mapa topográfico 3D correspondente ao hemisfério esquerdo; C: Mapa topográfico 3D correspondente a área posterior cerebral

A Figura 3 mostra o resumo da análise do sujeito 2, onde é descrito todas as áreas cerebrais que deveriam ser ativada (com base na Tabela 1) em cada questão dos testes de lógica, pré e pós treinamento, e as áreas que de fato foram ativadas pelo sujeito além das esperadas. 
CBIE-LACLO 2015

Anais do XXVI Simpósio Brasileiro de Informática na Educação (SBIE 2015)

\begin{tabular}{|c|c|c|c|c|}
\hline \multicolumn{3}{|c|}{ Coleta pré treinamento } & \multicolumn{2}{|c|}{ Coleta pós treinamento } \\
\hline \multicolumn{2}{|c|}{ Áreas que devem ser ativadas } & Áreas ativadas & Áreas que devem ser ativadas & Áreas ativadas \\
\hline \multirow{4}{*}{$\begin{array}{c}\text { Questão } \\
1\end{array}$} & Lobo Frontal & $\mathrm{x}$ & Lobo frontal direito & $x$ \\
\hline & Lobo temporal esquerdo & $\mathrm{x}$ & Lobo temporal esquerdo & \\
\hline & Lobo parietal & $x$ & Lobo parietal esquerdo & $\mathrm{x}$ \\
\hline & Lobo occipital & $x$ & Lobo occipital & $x$ \\
\hline \multirow{2}{*}{$\begin{array}{c}\text { Questão } \\
1\end{array}$} & & Lobo temporal direito & & Lobo temporal direito \\
\hline & & Sulco central direito & & Lobo parietal direito \\
\hline \multirow{4}{*}{$\begin{array}{c}\text { Questão } \\
2\end{array}$} & Lobo frontal esquerdo & & Lobo frontal & $x$ \\
\hline & Lobo temporal direito & $\mathrm{x}$ & Lobo temporal & $\mathrm{x}$ \\
\hline & Lobo parietal direito & $x$ & Lobo parietal direito & $x$ \\
\hline & Lobo occipital & $\mathrm{x}$ & Lobo occipital & $\mathrm{x}$ \\
\hline \multirow{4}{*}{$\begin{array}{l}\text { Questão } \\
2\end{array}$} & & Lobo frontal direito & & \\
\hline & & Lobo temporal esquerdo & & \\
\hline & & Lobo parietal esquerdo & & \\
\hline & & Sulco central direito & & \\
\hline \multirow{4}{*}{$\begin{array}{c}\text { Questão } \\
3\end{array}$} & Lobo frontal esquerdo & & Lobo frontal direito & $\mathrm{x}$ \\
\hline & Lobo temporal & $\mathrm{x}$ & Lobo temporal & $\mathrm{x}$ \\
\hline & Lobo parietal esquerdo & $x$ & Lobo parietal direito & $\mathrm{x}$ \\
\hline & Lobo occipital & $x$ & Lobo occipital & $x$ \\
\hline \multirow{3}{*}{$\begin{array}{c}\text { Questão } \\
3\end{array}$} & & Lobo frontal direito & & Lobo frontal esquerdo \\
\hline & & Lobo parietal direito & & Lobo parietal esquerdo \\
\hline & & Lobo central direito & & \\
\hline \multirow{4}{*}{$\begin{array}{c}\text { Questão } \\
4\end{array}$} & Lobo frontal & $x$ & Lobo frontal & $\mathrm{x}$ \\
\hline & Lobo temporal esquerdo & $\mathrm{x}$ & Lobo temporal direito & $\mathrm{x}$ \\
\hline & Lobo parietal & $x$ & Lobo parietal & $x$ \\
\hline & Lobo occipital & $x$ & Lobo occipital & $x$ \\
\hline \multirow{2}{*}{$\begin{array}{c}\text { Questão } \\
4\end{array}$} & & Lobo temporal direito & & \\
\hline & & Sulco central direito & & \\
\hline \multirow{4}{*}{$\begin{array}{c}\text { Questão } \\
5\end{array}$} & Lobo frontal esquerdo & & Lobo frontal direito & $x$ \\
\hline & Lobo temporal direito & $\mathrm{x}$ & Lobo temporal esquerdo & \\
\hline & Lobo parietal & $\mathrm{x}$ & Lobo parietal & $\mathrm{x}$ \\
\hline & Lobo occipital esquerdo & $\mathbf{x}$ & Lobo occipital direito & $\mathrm{x}$ \\
\hline \multirow{4}{*}{$\begin{array}{c}\text { Questão } \\
5\end{array}$} & & Lobo frontal direito & & Lobo frontal esquerdo \\
\hline & & Lobo temporal esquerdo & & Lobo temporal direito \\
\hline & & Lobo occipital direito & & Lobo occipital esquerdo \\
\hline & & Sulco central & & \\
\hline
\end{tabular}

Figura 3. Resumo das principais áreas cerebrais ativadas no sujeito 2 , durante os testes de lógica pré e pós treinamento. As células em amarelo correspondem as áreas que deveriam ser ativada em cada questão. $O$ " $X$ " indica que 0 indivíduo ativou a área correspondente. As células brancas correspondem as áreas ativadas não esperadas. As palavras "esquerdo" e "direito" indicam os hemisférios.

Na coleta pré treinamento (Figura 3), o sujeito apresentou maior atividade em grande parte das áreas esperadas para a resolução de cada questão. Porém, mostra alta atividade em áreas que não eram previstas para o desenvolvimento dos exercícios. Com isso, confirma-se a hipótese 1 , pois acredita-se que o sujeito tomou diversos caminhos até encontrar a possível solução do problema. No entanto, na coleta pós treinamento pode-se verificar que as áreas com maior atividade tiveram uma redução em comparação a coleta pré treinamento, confirmando assim a hipótese 2, pois o indivíduo, aparentemente, ativou um número menor de áreas não esperadas para resolver o problema e maior número de áreas necessárias para solucionar cada questão. 
CBIE-LACLO 2015

Anais do XXVI Simpósio Brasileiro de Informática na Educação (SBIE 2015)

\section{Conclusão}

O trabalho teve como objetivo investigar possíveis contribuições de treinamento de lógica computacional para o desenvolvimento de raciocínio lógico em estudantes do ensino médio. Para esse fim, foi necessário identificar possíveis mudanças na fisiologia cerebral pré e pós treinamento de lógica computacional, e ainda, caracterizar a atividade cerebral durante a realização de testes de lógica antes e depois do treinamento.

Tomando como referência o objetivo do estudo, pode ser considerado que o mesmo foi atingido de modo satisfatório. Comparando-se as hipóteses 1 e 2, observouse que houve alterações na fisiologia cerebral, confirmando assim ambas as hipóteses. Como uma primeira implicação dos resultados é possível afirmar que alunos ativaram maior número de áreas cerebrais antes de praticarem a lógica de programação.

O trabalho é inovador na área da computação a medida que são escassos os estudos no campo da lógica computacional que utilizem a captação de sinais cerebrais para analisar/acompanhar/monitorar as alterações (fisiológicas) durante o aprendizado de lógica de programação.

Ampliando o potencial dos achados, é possível também assumir que, o trabalho oferece subsídio para o desenvolvimento de outras pesquisas. Pensa-se que pode ser desenvolvido um estudo com maior tempo de treinamento, maior números de indivíduos para as coletas, e junto com o a aquisição dos sinais utilizar técnicas automáticas de mineração de dados para se encontrar resultados mais refinados, nos quais não poderem ser encontrados apenas com as imagens.

No entanto, no que diz respeito ao ensino (mesmo não sendo o foco principal deste trabalho), surgiram alguns questionamentos: é possível proporcionar esse ensino na educação básica? Estão os professores aptos a desenvolver essas competências na sala de aula? Diante desse cenário, há que se fomentar a revisão de currículos de uma formação docente, bem como a formação continuada, daquela que já atuam nas escolas.

\section{Referencias}

Carvalho, F. A. H. de; (2007) Reaprender a Aprender: a pesquisa como alternativa metacognitiva. Tese (Doutorado em Educação) ? Faculdade de Educação, Pontifícia Universidade Católica do Rio Grande do Sul, Porto Alegre.

Cosenza, R. and Guerra, L. (2011). Neurociência e Educação. Artmed, Porto Alegre.

Eysenck, M. W. and Keane, M. T. (2007). Manual de psicologia cognitive. Artmed, Porto Alegre.

Ferbellone, H. F. and Eberspacher, H. F. (2005). Lógica de programação: a construção de algoritmos e estruturas de dados. 3. Ed. Pearson Prentice Hall, São Paulo.

Gazzaniga, M. S. and Heatherton, T. (2005). Ciência psicológica: mente, cérebro e comportamento. Artmed, Porto Alegre.

Izquierdo, I. (2004).A arte de esquecer. Vieira e Lent, São Paulo.

Lent, R. (2001). Cem bilhões de neurônios: conceitos fundamentais de neu- 
CBIE-LACLO 2015

Anais do XXVI Simpósio Brasileiro de Informática na Educação (SBIE 2015)

rociência. Athenue. São Paulo.

Lent, R. (2008). Neurociência da Mente e do Comportamento.Guanabara Koogan, Rio de Janeiro.

Machado, S. and Cunha, M. and Velasques, B. and Minc, D. and Hugo, V. and Bastos, H. B. and Cagy, M. and Piedade, R. and Ribeiro, P. (2009). Interface cérebrocomputador: novas perpectivas para a reabilitação. Revista Neurociências, V. 17, N. 4, P. 329-335.

Silveira. J. A (2013). Análise de Sinais Cerebrais Utilizando Árvores de Decisão. Dissertação de mestrado, Universidade Federal do Rio Grande. Pós graduação em Modelagem Computacional.

Sternberg, R. J. (2012). Psicologia Cognitiva. Trad. Anna Maria Luche, Roberto Galman; Cengage Learning, São Paulo. 